\title{
Making the observational parsimonious richness a working mass proxy
}

\begin{abstract}
S. Andreon
INAF-Osservatorio Astronomico di Brera, via Brera 28, 20121 Milano, Italy

e-mail: stefano.andreon@brera.inaf.it

Received 12 March 2015 / Accepted 5 August 2015

ABSTRACT

Richness, i.e., the number of bright cluster galaxies, is known to correlate with the cluster mass, however, to exploit it as mass proxy we need a way to estimate the aperture in which galaxies should be counted that minimizes the scatter between mass and richness. In this work, using a sample of 39 clusters with accurate caustic masses at $0.1<z<0.22$, we first show that the scatter between mass and richness derived from survey data is negligibly small, as small as best mass proxies. The scatter turns out to be smaller than in some previous works and has a $90 \%$ upper limit of 0.05 dex in mass. The current sample, adjoining 76 additional clusters analyzed in previous works, establishes an almost scatterless, minimally evolving (if at all), mass-richness scaling in the redshift range $0.03<z<0.55$. We then exploit this negligible scatter to derive the reference aperture to be used to compute richness and to predict the mass of cluster samples. These predicted masses have a total 0.16 dex scatter with caustic mass, about half of which is not intrinsic to the proxy, but related to the noisiness of the caustic masses used for test proxy performances. These results make richness-based masses of best quality and available for large samples at a low observational cost.
\end{abstract}

Key words. galaxies: clusters: general - cosmological parameters

\section{Introduction}

In the next few years, numerous optical and near-infrared surveys, e.g., the Panoramic Survey Telescope and Rapid Response Systems (Kaiser et al. 2010), the Dark Energy Survey (Abbott et al. 2005), Large Synoptic Survey Telescope (Ivezic et al. 2008), Hyper-Suprime Camera (Takada 2010), and Euclid (Laureijs et al. 2011) are expected to generate galaxy catalogs over several thousands of square degrees to sufficient depth to reliably detect galaxy clusters up to $z \sim 2$. Indeed, the most distant cluster, JKCS 041 at $z=1.803$ (Andreon et al. 2009, 2014; Newman et al. 2014) has been discovered on the UKIRT infrared deep sky survey (Lawrence et al. 2007) and Euclid will reach deeper magnitudes over one thousand times wider fields (Laureijs et al. 2011).

One of the main goals of these surveys is to probe the expansion history of the Universe and place tight constraints on cosmological parameters using galaxy clusters. For example, accurate measurements of cluster abundance as a function of cluster mass and redshift can provide important constraints on cosmological parameters such as $\sigma_{8}$ and $w$ (see, e.g., Vikhlinin et al. 2009). However, the mass of a galaxy cluster is not directly measurable. These surveys will therefore rely on mass proxies and scaling relations between these observables and mass. Calibration of mass-observable scaling relations is therefore currently a high-priority observational goal because the use of galaxy clusters as cosmological probes is currently limited by our ability to relate observable properties and cluster mass through so-called observable-mass relations. For example, the major source of uncertainty in cosmological estimates by South Pole Telescope clusters is the currently available mass-proxy calibration, not sample size (Reichardt et al. 2013).
For photometric surveys, optical richness is the primary mass proxy, although stellar mass (i.e., the total cluster luminosity) has also been proposed as accurate alternative (Andreon 2012). The former has a scatter of 0.18 dex with mass (Andreon \& Hurn 2010, revised downward in Sect. 3.1), the latter has $90 \%$ upper limit scatter of 0.06 dex. These performances are however those achieved with knowledge of the radius in which galaxies have to be counted and are expected to degrade when this information is not available, i.e., when richness is used as a mass proxy. Indeed, richness has a 0.29 dex scatter with mass when $r_{200}$ is inferred from photometric data (Andreon \& Hurn 2010, revised downward in Sect. 3.2), which is much worse than if $r_{200}$ were known. Observables with large scatter are particularly problematic because of the sensitivity of cluster abundance on the uncertainty in the scatter of the mass-observable relation (Lima \& Hu 2005), and the latter are expected to increase with scatter. Consequently, a richness estimator that minimizes the scatter in the richnessmass relation is highly desirable to minimize the dilution of the cosmological information.

Therefore, an ideal mass proxy should be characterized by a low intrinsic scatter with cluster mass. It should also satisfy two more requirements. First, the proxy should be observationally parsimonious to obtain: low-scatter mass proxies are of little utility if they are unavailable because they require the acquisition of data challenging to obtain or their measurement is unfeasible. Second, the proxy should be relatively insensitive to the cluster dynamical state: a proxy that heavily relies on hydrostatic or dynamical equilibrium, as some X-ray mass observables, is of low utility for most of the clusters.

Once obtained, mass proxies need to be calibrated. Gravitational lensing offers a way to calibrate the richness-mass 
relation with the key advantage that the derived cluster mass does not rely on the assumption of hydrostatic or dynamical equilibrium. However, converting the lensing observable (reduced shear) into mass is challenging, as is nicely illustrated in detail by Hoekstra et al. (2015) and also is summarized in Andreon (2015a). Furthermore, the weak-lensing signal is also sensitive to mass in other structures "close" to the cluster (within say $1000 \mathrm{Mpc}$, because the weak-lensing signal changes little on these scales) such as large-scale-structure. By measuring the total mass projected along a long line of sight, lensing masses have an unavoidable minimal uncertainty of 20 to $50 \%$ (Meneghetti et al. 2010; Becker \& Kravtsov 2011) because of cluster asphericity and large-scale structure.

Caustics (Diaferio \& Geller 1997; Diaferio 1999) are also observationally demanding, because caustics rely on measurements of the escape velocity of galaxies, and are not affected by the cluster non-equilibrium, as weak lensing. Furthermore, and in contrast to the latter, caustics are not affected by the correlated large-scale structures along the line of sight (Diaferio 1999; Serra et al. 2011; Geller et al. 2013) because removing background/foreground at $\Delta v>3000 \mathrm{~km} \mathrm{~s}^{-1}$ is straightforward with spectroscopy that is needed anyway for caustics. This is magnificently illustrated by the cluster pair MS0906.5+1110/Abell 750: these clusters are offset by only $3000 \mathrm{~km} \mathrm{~s}^{-1}$ and are almost on the same line of sight. Caustics easily distinguish the two objects and two entries are found in Rines et al. (2013) mass catalog. Instead, lensing does not distinguish the two clusters and one single entry is found in Hoekstra et al. (2015) catalog, with mass given by the sum of the two clusters.

In this paper, we use an X-ray selected sample of 39 clusters with accurate caustic masses at $0.1<z<0.22$ to improve upon our past mass-richness calibration (Andreon \& Hurn 2010) and we show that the scatter between mass and richness derived from survey data is negligibly small, and indeed smaller than quoted in previous works. Our current analysis benefits of an improved understanding of caustic errors (Serra et al. 2011). We then exploit this negligible scatter to derive the reference aperture to be used to compute richness and to predict the mass of cluster samples, outperforming our (and other people) previous works. Finally, we compare the performances of richness and of a much advertised low-scatter mass proxy, the pseudo-pressure $Y_{S Z}$. The purpose of this work is not to find the optimal overdensity $\Delta$, which minimizes the scatter between predicted and true mass with a variable $\Delta$. Instead, we assumes that $M_{\Delta=200}$ is the target mass, and look at minimizing the scatter with this mass at this fixed overdensity.

Throughout this paper, we assume $\Omega_{\mathrm{M}}=0.3, \Omega_{\Lambda}=0.7$, and $H_{0}=70 \mathrm{~km} \mathrm{~s}^{-1} \mathrm{Mpc}^{-1}$. Magnitudes are in the $\mathrm{AB}$ system. We use the 2003 version of Bruzual \& Charlot (2003, hereafter BC03) stellar population synthesis models with solar metallicity, a Salpeter initial mass function (IMF) and a $z_{\mathrm{f}}=3$. Results of stochastic computations are given in the form $x \pm y$, where $x$ and $y$ are the posterior mean and standard deviation. The latter also corresponds to $68 \%$ intervals because we only summarized posteriors close to Gaussian in this way. All logarithms are in base 10 .

\section{Samples and data}

\subsection{The cluster sample}

Our starting point is the Hectospec Cluster Survey (HECS, Rines et al. 2013) cluster catalog. Clusters are: a) X-ray flux-selected; b) with $0.1<z<0.3$ (to allow a good caustic measurement);

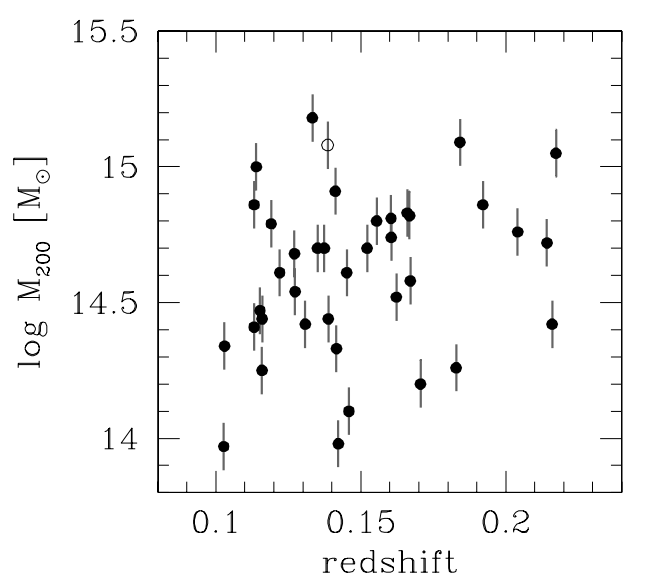

Fig. 1. Mass vs. redshift plot of the studied cluster sample. The open point is Abell 1068.

and c) in the SDSS DR6 footprint. The cluster catalog lists the cluster center, radius $r_{200}$, and mass within $r_{200}, M_{200}$, derived by the caustic technique ${ }^{1}$ (Diaferio \& Geller 1997; Diaferio 1999). These masses have, as mentioned, the advantage of not assuming the hydrostatic equilibrium or the relaxed status of the cluster and are computed using, on average, 177 member galaxies per cluster. These masses are three-dimensional (i.e., refer to the mass inside the sphere of radius $r_{200}$ ) and are derived assuming a spherical geometry. On the other hand, and similar to other mass estimates, the computation of their uncertainty is challenging (Serra et al. 2011; Gifford et al. 2013). Because of the finite sampling of the velocity field, these masses have a 20\% error (Serra et al. 2011). We adopt this figure, instead of the noisier and suspect (too small) values listed in Rines et al. (2013), and we account for the lack of precise knowledge of the true error, allowing the latter to be $95 \%$ of the times within a factor of 2 of the value derived from simulations (20\%) and marginalizing over this source of uncertainty (see Andreon \& Hurn 2010 concerning how to deal with noisy errors).

From the HECS cluster catalog we only keep clusters whose (passively evolved) limiting magnitude $M_{V, z=0}=-20 \mathrm{mag}$ is brighter than $r=20 \mathrm{mag}$, a conservative completeness value of the SDSS (Ivezic et al. 2002). This reduced the sample to clusters with $0.10<z \lesssim 0.22$, where the precise upper end depends on the value of the Galactic extinction in the cluster direction. From this sample, we only removed three clusters: a) the cluster pair MS0906.5+1110/Abell 750 because the richness of these clusters cannot be easily derived from photometry alone (see Fig. 11 in Geller et al. 2013) because they are almost on the same line of sight and separated by only $3000 \mathrm{~km} \mathrm{~s}^{-1}$; and b) the bimodal Abell 2055 cluster because it is formed by two clumps apart by about $r_{200}$. The spherical symmetry implicit in the caustic method is certainly not satisfied by this cluster, at least at the $r_{200}$ scale. Table 1 lists the 39 studied clusters, whose distribution in the redshift-mass plane is depicted in Fig. 1.

\subsection{The data and derivation of cluster richness}

Our analysis closely follows previous works, in particular Andreon \& Hurn (2010), which uses data from an earlier SDSS data release (for a different cluster sample), and

\footnotetext{
$r_{\Delta}$ is the radius within which the enclosed average mass density is $\Delta$ times the critical density at the cluster redshift.
} 

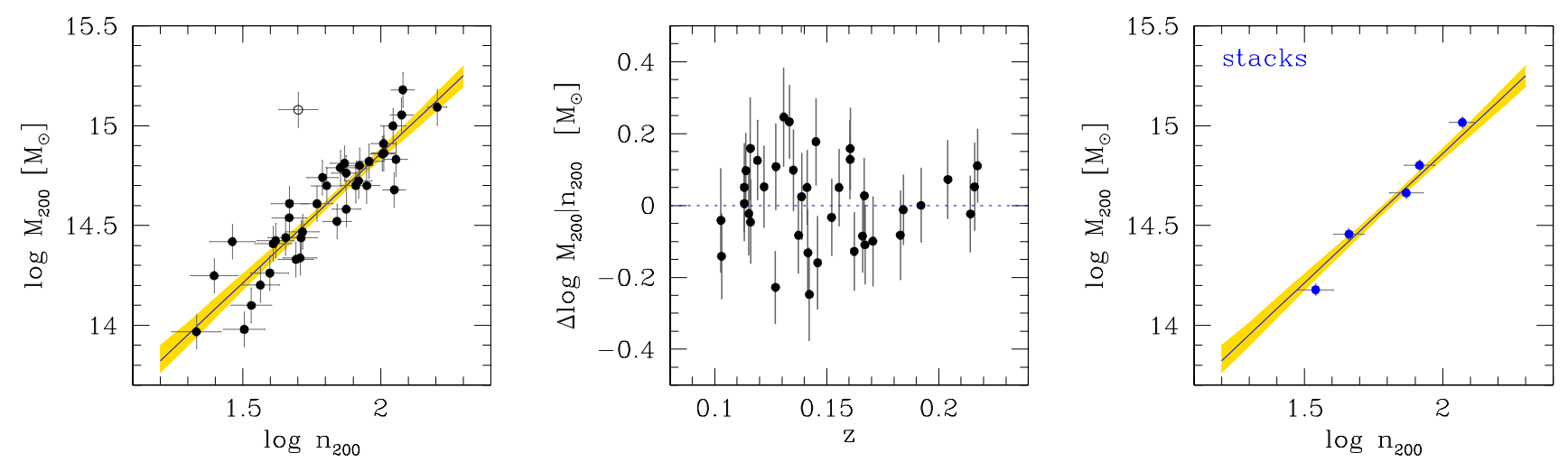

Fig. 2. Richness-mass scaling (left-hand and right-hand panels) and residuals (observed minus expected) as a function of redshift (central panel). The solid line marks the mean regression line ( of $\log M_{200}$ on $\log n_{200}$ ) fitted to the individual galaxy data, while the shading indicates the $68 \%$ uncertainty (highest posterior density interval). The right-hand panel combines clusters in stacks of eight clusters each, with the exception of the most massive point, composed of just six clusters. Masses are corrected for the negligible best-fit evolution. Points and approximated error bars are derived by adding errors summed in quadrature, as commonly done in the literature. The open point (Abell 1068) is not fitted and is out of scale in the central panel.

Andreon \& Congdon (2014), which extends the analysis to account for evolution of the mass-proxy scaling.

Basically, we aim to count red members within a specified luminosity range and color, and within the $r_{200}$ radius, as already done for other clusters (Andreon 2006, 2008; Andreon et al. 2008; Andreon \& Hurn 2010; Andreon \& Bergé 2012). For each cluster, we extracted the galaxy catalogs from the Sloan Digital Sky Survey (SDSS) 12th data release (Alam et al. 2015). Total galaxy magnitudes refer to "cmodel", while colors are based on "model" magnitude. Colors are corrected for the color-magnitude slope, although this is a minor correction given the small magnitude range explored. We adopt a simple definition of "red", by only counting galaxies within 0.1 redward and 0.2 blueward in $g-r$ of the color-magnitude relation.

Some of the red galaxies in the cluster line of sight are actually in the cluster fore/background. The contribution from background galaxies is estimated, as usual, from a reference direction (e.g., Zwicky 1957; Oemler 1974; Andreon et al. 2005). The reference direction is formed of three octants, free of contaminating structures (other clusters) and not badly affected by the SDSS imaging masks, of a corona centered on the studied cluster with inner radius $3 \mathrm{Mpc}$ and outer radius 1 degree, therefore fully guaranteeing homogeneous data for cluster and control field. The precise background estimation is however of secondary importance: we found that if a single redshiftdependent value of the background counts per unit $\operatorname{arcmin}^{2}$ were used for all clusters, indistinguishable results would be obtained.

The derived (projected) richness values are listed in Table 1. Since richness is based on galaxy counts, it is computed within a cylinder of radius $r_{200}$. The mean (log) richness error is 0.06 dex. Richness errors account for Poisson fluctuations in background+cluster counts and the uncertainty in the mean background counts, as detailed in previous works (e.g., Andreon \& Congdon 2014; Andreon \& Hurn 2010). The derivation of these errors assumes a Gaussian approximation, which is only partially satisfied for these data. For this reason, we also list raw galaxy counts in the cluster and control field directions, and the ratio of the solid angles in which they are computed. These values are used for the mass-richness fit, therefore removing the Gaussian approximation of the listed $n_{200}$ error.

\section{Results}

\subsection{The mass-richness scaling}

Following previous works, we fit the data with a linear relation on log-quantities:

$\log M_{200}=\alpha+\beta\left(\log n_{200}-2.0\right)+\gamma \log \frac{1+z}{1.15}$

allowing for an intrinsic scatter $\sigma_{\text {intr }}$, noisy mass errors and adopting pivot values of richness and $1+z$ close to the sample mean (the values 2.0 and 1.15 in Eq. (1)). We adopt weak priors on parameters (see Andreon \& Hurn 2010 for details). To account for the noisiness of mass errors, our baseline analysis uses $v=6$ to quantify that we are $95 \%$ confident that quoted mass errors are correct up to a factor of 2 (as mentioned) and we anticipate that results are robust to the choice of $v$. Since all clusters in the sample have a measured richness, there is no selection function (at a given mass) to account for (see Andreon \& Bergé 2012; Andreon \& Congdon 2014). We choose not to account for the data structure (the mass function and the induced Malmquist-Eddington correction in the astronomical parlance, see Andreon \& Bergé 2012 for details) because we have a limited aim in this work: we acknowledge that results are derived, and only valid, for clusters drawn from an X-ray selected sample in a given redshift range ${ }^{2}$.

The fit to mass, richness, and redshift is shown in Fig. 2. Abell 1068 (the open point in Fig. 2) is an outlier. This cluster is also an outlier of the $L_{X}$-mass relation (Rines et al. 2013), and we checked that it is also an outlier in the relation between caustic mass and the X-ray mass given in Piffaretti et al. (2011). We therefore removed this cluster from the fitting and analysis, although we show it (with a different symbol) in the figures. For display purposes only, the right-hand panel shows a binned version of the left-hand panel, by combining clusters in bins of eight clusters each, except the most massive one formed by six clusters.

Fitting the individual values, we found that richness scales almost linearly with mass (with slope $s=1.30 \pm 0.10$ ), with a

2 This statement is overly restrictive, see Andreon (2015b) for details. 

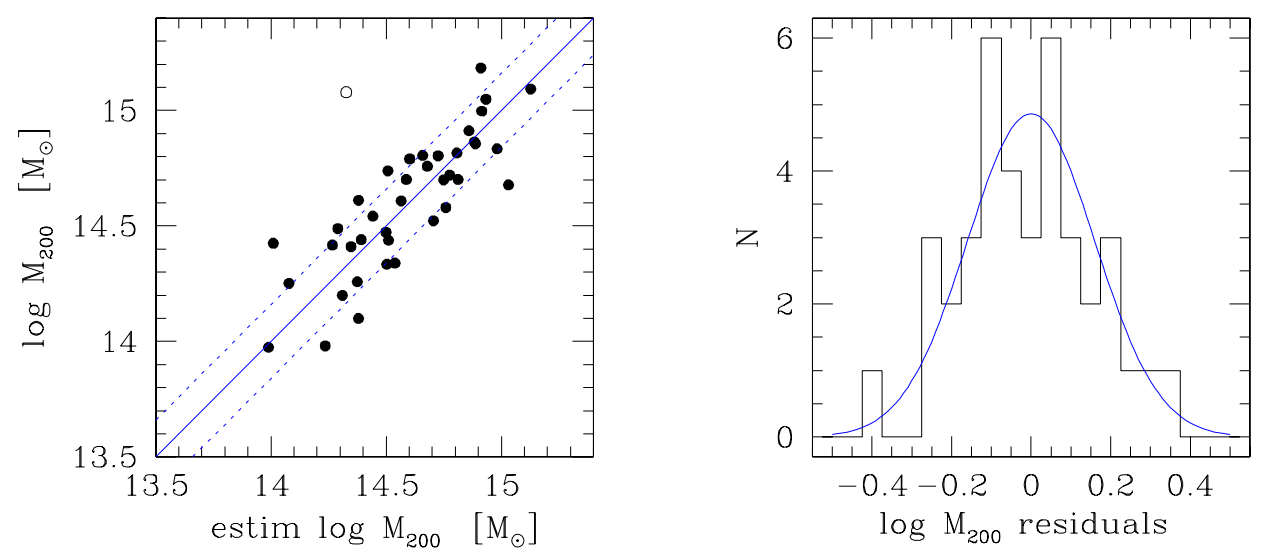

Fig. 3. Performances of the richness proxy (i.e., when the true $r_{200}$ is unknown). Left-hand panel: mass-predicted from richness vs. true (caustic) mass. The solid line is the one-to-one relation (not a fit to the data). The dotted lines show the above line plus or minus the data scatter (0.16 dex). The open point marks the outlier Abell 1068 cluster. Right-hand panel: mass residuals (predicted minus true) with superposed a Gaussian with $\sigma=0.16$ dex.

negligible redshift-dependent term (slope $-0.1 \pm 1.0$ ), and with a negligible intrinsic scatter. More precisely,

$$
\begin{aligned}
\log M_{200}= & 14.86 \pm 0.03+(1.30 \pm 0.10)\left(\log n_{200}-2.0\right) \\
& +(-0.1 \pm 1.0) \log \frac{1+z}{1.15}
\end{aligned}
$$

The evolution of the richness-mass relation is poorly determined because of the reduced redshift baseline $(\Delta z=0.1)$, compared to the more accurate determination in Andreon \& Congdon (2014) benefiting from a much larger redshift baseline, $0.15<z<0.55$, but using richness and mass measured within a fixed metric aperture.

The intrinsic scatter is, as mentioned, negligibly small: the amplitude of the intrinsic scatter is set by the difference of the data scatter and measurement errors. The former is 0.12 dex, the latter are $\sim 0.08$ dex on both axis, giving little or no room for an additional intrinsic scatter. In fact, our Bayesian analysis found an intrinsic scatter of $\sigma_{\log M_{200} \mid n_{200}}<0.05$ dex with $90 \%$ probability, consistent and more precise than the value found using weak-lensing masses in Andreon \& Congdon (2014). The negligible intrinsic scatter between richness (measured in cylinders) and mass (measured in spheres) indicates a negligible effect of large-scale-structure on the richness proxy, unless it is strongly covariant with cluster elongation along the line of sight. The found tight richness-mass scaling is one of the two main results of this work.

The negligible scatter of the richness-mass scaling (posterior mean: $0.02 \mathrm{dex}$ ) scores well compared to those of best surveybased mass proxies: stellar mass (0.08 dex, Andreon 2012, based however on a reduced cluster sample), $Y_{\text {sph }}$ (0.09 dex, Marrone et al. 2012), and Planck $Y_{S Z}(>0.06$ dex, Planck Collaboration XI 2011). The negligible scatter of $n_{200}$ also fares well compared to proxies that requires follow-up observations, such as gas mass $(\sim 0.06$ dex, Mahdavi et al. 2013$)$ or pseudo-pressure $Y_{X}(\sim 0.04$ to $\sim 0.10$ dex, Arnaud et al. 2007; Mahdavi et al. 2013).

We improve upon our previous work (Andreon \& Hurn 2010), based on the CIRS sample of Rines et al. (2006), by reducing the uncertainties of intercept and slope by $30 \%$, and the uncertainty of intrinsic scatter by a $>3$ factor as a result of better understood errors and better determined cluster centers (especially important when determining the intrinsic scatter, see also Andreon 2012). Unfortunately, these two samples cannot be easily combined because they adopt slightly different definitions of $M_{200}$.
To summarize, the 39 clusters studied in this work and the 23 massive clusters at $0.15<z<0.55$ in Andreon \& Congdon (2014) agree in establishing an almost scatterless, minimally evolving (if at all), mass-richness scaling, in broad agreement with the 53 clusters at lower redshift studied in Andreon \& Hurn (2010).

\subsection{An improved mass proxy estimate}

The tightness of the richness-mass scaling makes richness an interesting mass proxy. However, as remarked several times, but not always appreciated, if the observable itself requires knowledge of the cluster mass (because knowledge of $r_{200}$ is needed) such an observable is not ready to be used as mass proxy until a way to estimate $r_{200}$ is found. In this section we make richness an effective mass proxy by finding a way to estimate $r_{200}$ that minimally degrades the performances of the mass proxy.

In Andreon \& Hurn (2010) we measured the cluster richness within a fixed aperture, the relation between $r_{200}$ and this (aperture) richness is calibrated with real data and used to infer the aperture radius, $\widehat{r_{200}}$, in which richness should be finally computed (and mass estimated). Because of the inherent scatter in the individual radial profiles of galaxy clusters between aperture radius and $\widehat{r_{200}}$, a large amount of scatter is introduced in the mass-proxy scaling.

Kravtsov et al. (2006) proposed a different approach for the $Y_{X}$ mass proxy, later also adopted for the $Y_{S Z}$ proxy (Planck Collaboration XXVII 2015) and hereafter for richness: $r_{200}$ and the proxy value ( $n_{200}$ in our case) are estimated at the same time assuming that the proxy-mass relation is scatterless. In practice, an iterative approach is taken: a radius $r$ is taken $(1.4 \mathrm{Mpc}$ in our case), $n(<r)$ estimated, then $r$ is updated to the value appropriate for the derived richness if the mass-richness were scatterless (i.e., using Eq. (2), and noting that $r_{200}=M_{200}^{1 / 3}$ apart from obvious coefficients) and then the process is iterated until convergence. In our case, judging from comparison with results obtained after ten iterations, convergence is achieved at the second or third iteration. We therefore adopt the fifth iteration as the final (but any other iteration would give equivalent results). These values of richness and masses, derived without knowledge of the true $r_{200}$, are listed in Table 1.

The left-hand panel of Fig. 3 shows the performance of the richness proxy when ready to be used for cluster samples of 
Table 1. Cluster sample and results of the analysis.

\begin{tabular}{|c|c|c|c|c|c|c|c|c|}
\hline Cluster id & (2) & $\begin{array}{r}\log M_{200} \\
{\left[M_{\odot}\right]} \\
(3) \\
\end{array}$ & $n_{200}$ & obstot & obsbkg & $(7)$ & $\begin{array}{r}\log \widehat{M}_{200} \\
{\left[M_{\odot}\right]} \\
(8) \\
\end{array}$ & $\widehat{n_{200}}$ \\
\hline Zw1478 & 0.103 & 13.97 & $21 \pm 5$ & 24 & 43 & 16.940 & 13.99 & $21 \pm 5$ \\
\hline A 1235 & 0.103 & 14.34 & $51 \pm 8$ & 58 & 68 & 9.733 & 14.54 & $57 \pm 8$ \\
\hline A2034 & 0.113 & 14.86 & $102 \pm 11$ & 114 & 68 & 5.422 & 14.89 & $108 \pm 11$ \\
\hline Zw8197 & 0.113 & 14.41 & $41 \pm 7$ & 47 & 67 & 10.760 & 14.35 & $40 \pm 7$ \\
\hline A2069 & 0.114 & 15.00 & $111 \pm 13$ & 148 & 164 & 4.425 & 14.92 & $110 \pm 12$ \\
\hline A1302 & 0.115 & 14.47 & $52 \pm 8$ & 58 & 60 & 10.140 & 14.50 & $53 \pm 8$ \\
\hline A1361 & 0.116 & 14.25 & $25 \pm 6$ & 30 & 74 & 14.430 & 14.08 & $25 \pm 5$ \\
\hline A1366 & 0.116 & 14.44 & $51 \pm 8$ & 57 & 60 & 10.860 & 14.51 & $54 \pm 8$ \\
\hline A2050 & 0.119 & 14.79 & $71 \pm 9$ & 83 & 77 & 6.689 & 14.60 & $63 \pm 9$ \\
\hline A1033 & 0.122 & 14.61 & $59 \pm 8$ & 68 & 86 & 9.306 & 14.57 & $59 \pm 8$ \\
\hline A655 & 0.127 & 14.68 & $112 \pm 11$ & 120 & 73 & 9.113 & 15.03 & $135 \pm 12$ \\
\hline A646 & 0.127 & 14.54 & $47 \pm 7$ & 54 & 83 & 11.250 & 14.44 & $48 \pm 7$ \\
\hline A1930 & 0.131 & 14.42 & $29 \pm 6$ & 36 & 100 & 14.250 & 14.01 & $22 \pm 5$ \\
\hline A1437 & 0.133 & 15.18 & $120 \pm 12$ & 144 & 109 & 4.614 & 14.91 & $110 \pm 11$ \\
\hline A1132 & 0.135 & 14.70 & $64 \pm 9$ & 76 & 122 & 9.948 & 14.59 & $62 \pm 9$ \\
\hline A795 & 0.137 & 14.70 & $89 \pm 10$ & 101 & 123 & 10.290 & 14.81 & $92 \pm 10$ \\
\hline A1068 & 0.139 & 15.08 & $50 \pm 9$ & 76 & 151 & 5.865 & 14.33 & $39 \pm 7$ \\
\hline A1918 & 0.139 & 14.44 & $45 \pm 7$ & 53 & 122 & 15.680 & 14.39 & $44 \pm 7$ \\
\hline A1413 & 0.141 & 14.91 & $103 \pm 11$ & 120 & 137 & 7.865 & 14.86 & $100 \pm 11$ \\
\hline A990 & 0.142 & 14.33 & $49 \pm 8$ & 60 & 206 & 19.220 & 14.50 & $53 \pm 8$ \\
\hline Zw3179 & 0.142 & 13.98 & $32 \pm 6$ & 36 & 133 & 33.320 & 14.24 & $33 \pm 6$ \\
\hline A667 & 0.145 & 14.61 & $47 \pm 8$ & 57 & 136 & 13.200 & 14.38 & $43 \pm 7$ \\
\hline A1978 & 0.146 & 14.10 & $34 \pm 6$ & 40 & 177 & 29.230 & 14.38 & $43 \pm 7$ \\
\hline A2009 & 0.152 & 14.70 & $81 \pm 10$ & 94 & 161 & 12.630 & 14.75 & $82 \pm 10$ \\
\hline A980 & 0.155 & 14.80 & $84 \pm 10$ & 101 & 192 & 11.220 & 14.73 & $79 \pm 10$ \\
\hline RXJ1720 & 0.160 & 14.81 & $74 \pm 10$ & 89 & 179 & 11.890 & 14.66 & $70 \pm 9$ \\
\hline A2259 & 0.160 & 14.74 & $61 \pm 9$ & 75 & 178 & 13.180 & 14.51 & $54 \pm 8$ \\
\hline A1902 & 0.162 & 14.52 & $69 \pm 9$ & 77 & 142 & 18.780 & 14.71 & $76 \pm 9$ \\
\hline A1914 & 0.166 & 14.83 & $114 \pm 11$ & 126 & 150 & 12.160 & 14.98 & $124 \pm 12$ \\
\hline A1553 & 0.167 & 14.82 & $91 \pm 10$ & 104 & 167 & 12.610 & 14.81 & $91 \pm 10$ \\
\hline A1201 & 0.167 & 14.58 & $75 \pm 9$ & 89 & 254 & 18.180 & 14.76 & $84 \pm 10$ \\
\hline A1204 & 0.171 & 14.20 & $37 \pm 6$ & 41 & 149 & 33.860 & 14.31 & $38 \pm 7$ \\
\hline A2187 & 0.183 & 14.26 & $40 \pm 7$ & 46 & 225 & 35.260 & 14.38 & $42 \pm 7$ \\
\hline A1689 & 0.184 & 15.09 & $160 \pm 13$ & 180 & 193 & 9.919 & 15.13 & $160 \pm 14$ \\
\hline A1246 & 0.192 & 14.86 & $103 \pm 11$ & 121 & 277 & 15.240 & 14.88 & $104 \pm 11$ \\
\hline A963 & 0.204 & 14.76 & $75 \pm 9$ & 89 & 282 & 20.020 & 14.68 & $73 \pm 9$ \\
\hline A 1423 & 0.214 & 14.72 & $83 \pm 10$ & 97 & 321 & 23.120 & 14.78 & $87 \pm 10$ \\
\hline Zw2701 & 0.216 & 14.42 & $42 \pm 7$ & 48 & 239 & 37.400 & 14.27 & $35 \pm 6$ \\
\hline A773 & 0.217 & 15.05 & $119 \pm 12$ & 148 & 413 & 14.330 & 14.93 & $114 \pm 12$ \\
\hline
\end{tabular}

Notes. The table lists cluster id, redshift $z$, mass $M_{200}$ from Rines et al. (2012), followed by values derived in this work: richness $n_{200}$, observed galaxy counts in the cluster and control field directions ( $o b s t o t$ and $o b s b k g$ ), their solid angle ratios $C$. Columns eight and nine give the predicted mass $\widehat{M_{200}}$ and richness $\widehat{n_{200}}$ we derived only using richness. Masses are within spheres, richnesses within cylinders. Masses in Col. (3) have 0.08 dex errors, those in Col. (8) have a 0.16 dex total error. Cluster coordinates are in Rines et al. (2012).

unknown mass (i.e. without knowledge of the true $r_{200}$ ): the richness in the estimated $r_{200}$, converted in mass using Eq. (2), i.e., the richness-predicted mass, is plotted versus the caustic mass. The scatter between the richness-predicted mass and mass is $0.16 \pm 0.02 \mathrm{dex}$ (error computed by bootstrapping), also shown in the right-hand panel of Fig. 3. Of this, 0.08 dex is due to the noisiness of the caustic mass estimate (i.e., caustic errors).

Our quoted error of the richness-based mass is total, in contrast to what is quoted for other mass estimates. For example, it includes the scatter around the relation and errors on the observable. Our choice of quoting all known error terms in the error budgets is not widespread: for example the error quoted by the Planck team for the $Y_{S Z}$-based masses does not include the terms above (Sect. 5.3 of Planck Collaboration XXVII 2015). Finally, and in contrast to some other claims about the error of other proxies, the scatter we derived above is not computed assuming we know the true $r_{200}$. If we had to follow these works, we should claim a $\sim 0.02$ dex error, not the quoted 0.16 dex error.

As mentioned, we removed one single cluster pair (Abell 750/MS0906.5+1110) out of the starting sample (about 40 clusters) because one is projected on the top of the other and with $\Delta v \sim 3000 \mathrm{~km} \mathrm{~s}^{-1}$, leading to an erreneous richness (and lensing mass). This situation is very rare, no other cluster has been removed from the sample for richness-based reasons, and the other two removed clusters (Abell 1068 and Abell 2055) have unproblematic richnesses but questionable caustic masses. If this sample is representative of clusters in the Universe, we then expect that richness-based masses will be very wrong for only about $2 \%$ of the clusters.

The above predicted mass uses accurate cluster centers. Since we also want to exploit richness as mass proxy for clusters without accurate centers, we now check the impact of lacking 

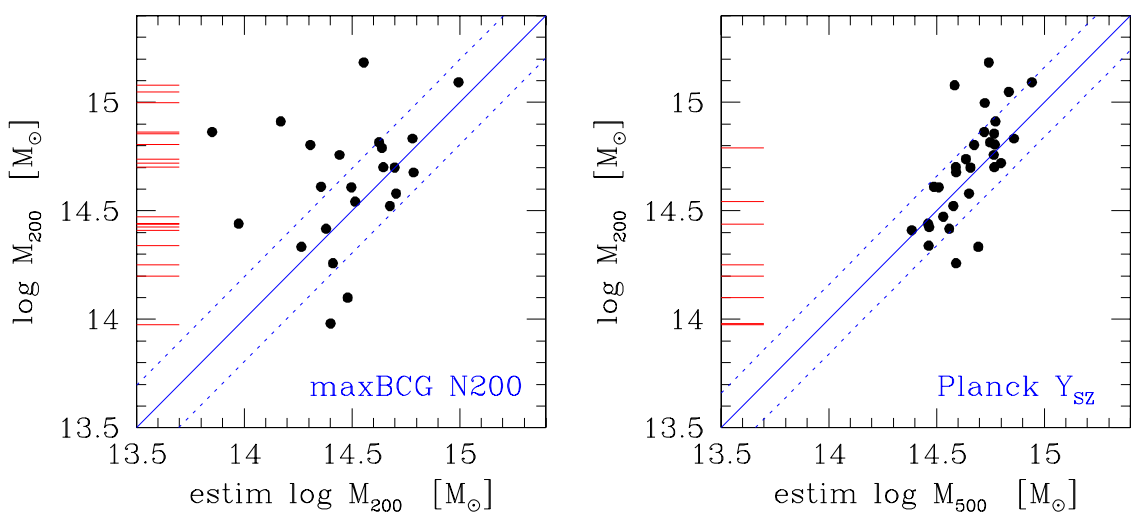

Fig. 4. Performances of maxBCG richness (left-hand panel) and of Planck $Y_{S Z}$ (right-hand panel) proxies. The plots compare the richness- or $Y_{S Z}$-predicted masses vs. the caustic mass for the very same sample used in Fig. 3. The solid line is the one-to-one relation (not a fit to the data). The dotted lines show the above line plus or minus the claimed mass precision (left-hand panel) or our richness performances (right-hand panel) for comparison with Fig. 3. The (red) ticks on the ordinate of the three panels indicates unmatched clusters.

accurate centers. We therefore implement a way to estimate centers. We iteratively derive the cluster center only using galaxy counts: we consider an aperture of $1.0 \mathrm{Mpc}$ radius, we compute the median right ascension and declination, we consider this the new center, and we iterate ten times. We start the center computation 3 arcmin, about $0.5 \mathrm{Mpc}$ at the median redshift, away from the true cluster center to avoid the advantage of starting from the optimal center. We take the latest computed centers as new cluster centers (but results would be unchanged using, e.g., the fifth iteration), and we solve for radius and mass as before. We found an identical scatter between the richness-predicted mass and mass, as expected because the center error is negligible compared to $r_{200}$.

Our current richness scores best among all mass proxies based on survey data, as far as we are aware of, as we now illustrate.

First, the left-hand panel of Fig. 4 shows the performance of the maxBCG richness using the mass calibration in Rozo et al. (2009) via plotting the richness-predicted mass vs. caustic mass for the very same HECS sample shown in Fig. 3. While using similar photometry (a previous SDSS release), these authors adopt different recipes for counting the galaxies (notably the adopted aperture) and a different (indirect) approach to mass calibration. Clusters are matched with a 2 arcmin maximum radius and our results are robust to adopted matching aperture. Clusters not found in the maxBCG catalog (about 40\%) are shown as ticks on the ordinate. For the remaining $60 \%$, we found a scatter of 0.26 dex (consistent with, but larger than, the advertised value). Our mass proxy performs better, both in terms of scatter and in terms of completeness. Adopting the more recent calibration by Rozo et al. (2014) does not change our results. By taking the richest maxBCG cluster with consistent redshift and within a large matching radius does not change our results either. The improved maxBCG richness by Rykoff et al. (2012) shows a reduced scatter (although larger than advertised) and a large mass bias, the latter anticipated by the authors because of their preliminary calibration. By adopting a direct approach to mass calibration, our richness is not badly affected by systematics. More recent catalogs based on SDSS lack a mass calibration. Considering them would require us to calibrate them first, which is beyond the purpose of this paper.

Second, the right-hand panel of Fig. 4 illustrates the performances of $Y_{S Z}$-based masses, taken from Planck Collaboration XXVII (2015) via plotting the $Y_{S Z}$-predicted mass vs. caustic mass. Clusters are matched with a maximal radius of 3 arcmin (equal to about twice the $68 \%$ Planck position error). Unmatched clusters (eight cases) are marked with red tick on the figure ordinate. Some of them are of low mass and are therefore likely absent in the Planck catalog because they are undetected. However, some of them are somewhat massive. The scatter of the remaining $31 Y_{S Z}$-based masses is comparable to richness-based masses ( 0.18 vs. $0.16 \mathrm{dex})$, but perhaps better described by a combination of a slightly narrower distribution (with $\sigma \sim 0.14$ dex) plus some (15\%) outliers. Enlarging the matching radius does not help to increase the $Y_{S Z}$ mass performances because the newly matched objects have large scatter with mass. Decreasing the matching radius is of no help either because it decreases the number of matches without reducing the scatter. By restricting our attention to clusters in the narrow redshift range studied in this work $(0.10<z<0.22)$ and by computing the minimal scatter (by allowing a free mass bias), we minimize known redshift- (Andreon 2014) and bias(von der Linden et al. 2014; Planck Collaboration XXIV 2015) effects of $Y_{S Z}$-based masses. To summarize our comparison with $Y_{S Z}$-based masses, proxy availability and a low intrinsic scatter with mass are essential to evaluate the quality of a mass proxy. The 54 s exposures taken at a $2 \mathrm{~m}$ telescope in a mediocre site on Earth (the SDSS) competes well with a satellite at the L2 point from this point of view in the studied redshift range ${ }^{3}$.

Third, our score of a 0.16 dex scatter is better than all 25 methods considered in the mass reconstruction project (Old et al. 2015), most of which requires spectroscopy, i.e., are observationally more demanding. In particular, in these simulations richness-based masses outperform caustic masses. If the same holds true in our Universe, our work would be measuring the scatter of a precise mass estimate (richness) via comparison to a noisy estimate (caustic masses)! Note however that such a simulation-based analysis should be considered with caution because results depends on the way clusters are populated by galaxies in the simulations, and because nothing guarantee that the best mass proxy in simulations is also best in our Universe.

We recal that a tight mass-proxy scaling is certainly useful, but scalings that involve observationally parsimonious

\footnotetext{
3 One may argue that we favored optical richness by removing the cluster pair MS0906.5+1110/Abell 750 from the sample. However, $Y_{S Z}$-based masses suffer of the same alignment problem because the $Y_{S Z}$ observable lacks almost any redshift sensitivity.
} 
observables are even better, and richness is both parsimonious and display a tight relation with mass. Indeed, richness has already allowed us to derive the mass of three among the most distant clusters and groups known (Strazzullo et al. 2013; Andreon et al. 2014; Webb et al. 2015), which still miss $Y_{X^{-}}$or $Y_{S Z}$-based masses because they are observationally expensive to acquire.

Cluster counts are exponentially sensitive to the properties of dark energy and to the square of the scatter between proxy and mass. Our factor two improvement over Andreon \& Hurn (2010) and maxBCG, obtained because the current estimate benefits from a radius measured very close to the true $r_{200}$ and tailored to each cluster, corresponds to a four times lower dilution of cosmological information contained in number counts. This improvement is our second main result.

\section{Conclusions}

We studied $39 \mathrm{X}$-ray selected clusters at $0.1<z<0.22$ with accurate caustic masses (20\% errors) from the HECS catalog. We derived richness within $r_{200}$ using SDSS photometry and we found an extremely tight, almost scatterless, richness-mass scaling, at least as tight as those derived for other mass proxies, and tighter than quoted in previous works. The current sample, adjoinining 76 more clusters analyzed in previous works, establishes an almost scatterless, minimally evolving (if at all), mass-richness scaling in the redshift range $0.03<z<0.55$. By assuming that the true relation is scatterless, we rederive cluster richness and $r_{200}$ at the same time only using SDSS photometry. We found that the newly derived richness is a mass proxy with 0.16 dex scatter, half of which is not intrinsic to the proxy, but related to the noisiness of the caustic masses used for test richness performances.

To summarize, an ideal mass proxy should be characterized by a low intrinsic scatter, be observationally parsimonious to obtain, relatively insensitive to the cluster dynamical state, and easy to compute. Richness seems to be as such. For this reason we are now computing richness-predicted masses of many $z<0.22$ clusters in the SDSS footprint, with priorities set by the community input.

Acknowledgements. This work benefits of discussion with caustic specialists, Antonaldo Diaferio, Ana Laura Serra, and Jacob Svensmark, and with Andrea Biviano.

\section{References}

Abbott, T., Abdalla, F. B., Allam, S., et al., 2005, ArXiv e-prints [arXiv:astro-ph/0510346]

Alam, S., Albareti, F. D., Allende Prieto, C., et al. 2015, ApJS, 219, 12
Andreon, S. 2006, MNRAS, 369, 969

Andreon, S. 2008, MNRAS, 386, 1045

Andreon, S. 2012, A\&A, 548, A83

Andreon, S. 2014, A\&A, 570, L10

Andreon, S. 2015a, A\&A, 575, A108

Andreon, S. 2015b, A\&A, submitted

Andreon, S., \& Bergé, J. 2012, A\&A, 547, A117

Andreon, S., \& Congdon, P. 2014, A\&A, 568, A23

Andreon, S., \& Hurn, M. A. 2010, MNRAS, 404, 1922

Andreon, S., Punzi, G., \& Grado, A. 2005, MNRAS, 360, 727

Andreon, S., Puddu, E., de Propris, R., \& Cuillandre, J.-C. 2008, MNRAS, 385, 979

Andreon, S., Maughan, B., Trinchieri, G., \& Kurk, J. 2009, A\&A, 507, 147

Andreon, S., Newman, A. B., Trinchieri, G., et al. 2014, A\&A, 565, A120

Arnaud, M., Pointecouteau, E., \& Pratt, G. W. 2007, A\&A, 474, L37

Becker, M. R., \& Kravtsov, A. V. 2011, ApJ, 740, 25

Bruzual, G., \& Charlot, S. 2003, MNRAS, 344, 1000

Diaferio, A. 1999, MNRAS, 309, 610

Diaferio, A., \& Geller, M. J. 1997, ApJ, 481, 633

Geller, M. J., Diaferio, A., Rines, K. J., \& Serra, A. L. 2013, ApJ, 764, 58

Gifford, D., Miller, C., \& Kern, N. 2013, ApJ, 773, 116

Ivezić, Ž., Menou, K., Knapp, G. R., et al. 2002, AJ, 124, 2364

Ivezic, Z., Axelrod, T., Brandt, W. N., et al. 2008, Serb. Astron. J., 176, 1

Kaiser, N., Burgett, W., Chambers, K., et al. 2010, Proc. SPIE, 7733

Kravtsov, A. V., Vikhlinin, A., \& Nagai, D. 2006, ApJ, 650, 128

Laureijs, R., Amiaux, J., Arduini, S., et al. 2011, ArXiv e-prints [arXiv: 1110.3193]

Lawrence, A., Warren, S. J., Almaini, O., et al. 2007, MNRAS, 379, 1599

Mahdavi, A., Hoekstra, H., Babul, A., et al. 2013, ApJ, 767, 116

Marrone, D. P., Smith, G. P., Okabe, N., et al. 2012, ApJ, 754, 119

Meneghetti, M., Rasia, E., Merten, J., et al. 2010, A\&A, 514, A93

Newman, A. B., Ellis, R. S., Andreon, S., et al. 2014, ApJ, 788, 51

Oemler, A., Jr. 1974, ApJ, 194, 1

Old, L., Wojtak, R., Mamon, G. A., et al. 2015, MNRAS, 449, 1897

Piffaretti, R., Arnaud, M., Pratt, G. W., Pointecouteau, E., \& Melin, J.-B. 2011, A\&A, 534, A109

Planck Collaboration XI. 2011, A\&A, 536, A11

Planck Collaboration XXIV. 2015, A\&A, submitted [arXiv: 1502.01597 ]

Planck Collaboration XXVII. 2015, A\&A, in press, DOI: $10.1051 / 0004-6361 / 201525823$

Reichardt, C. L., Stalder, B., Bleem, L. E., et al. 2013, ApJ, 763, 127

Rines, K., \& Diaferio, A. 2006, AJ, 132, 1275

Rines, K., Geller, M. J., Diaferio, A., \& Kurtz, M. J. 2013, ApJ, 767, 15

Rozo, E., Rykoff, E. S., Evrard, A., et al. 2009, ApJ, 699, 768

Rozo, E., Bartlett, J. G., Evrard, A. E., \& Rykoff, E. S. 2014, MNRAS, 438, 78

Rykoff, E. S., Koester, B. P., Rozo, E., et al. 2012, ApJ, 746, 178

Serra, A. L., Diaferio, A., Murante, G., \& Borgani, S. 2011, MNRAS, 412, 800

Strazzullo, V., Gobat, R., Daddi, E., et al. 2013, ApJ, 772, 118

Takada, M. 2010, AIP Conf. Ser., 1279, 120

Vikhlinin, A., Kravtsov, A. V., Burenin, R. A., et al. 2009, ApJ, 692, 1060

von der Linden, A., Mantz, A., Allen, S. W., et al. 2014, MNRAS, 443, 1973

Webb, T., Noble, A., DeGroot, A., et al. 2015, ApJ, 809, 173

Zwicky, F., Herzog, E., Wild, P., Karpowicz, M., \& Kowal, C. T. 1961, Catalogue of Galaxies and of Clusters of galaxies, Vol. I (Pasadena: California Institute of Technology, CIT) 